\title{
Leber's Hereditary Optic Neuropathy as a Promising Disease for Gene Therapy Development
}

\author{
Cuneyt Karaarslan
}

Received: August 26, 2019 / Published online: October 11, 2019

(C) The Author(s) 2019

\begin{abstract}
Leber's hereditary optic neuropathy (LHON) is a relatively common, rapidly progressing inherited optic neuropathy wherein LHON-affected eyes undergo optic nerve atrophy due to retinal ganglion cell (RGC) loss. It is a maternally inherited (or sporadic) mitochondrial disorder caused primarily by mutations in genes that encode components of respiratory complex (RC) 1 in mitochondria. Mitochondrial deficiency of RC1 compromises ATP production and oxidative stress management in RGCs. The most common LHON-causing mutations are $11778 \mathrm{G}>\mathrm{A}$, $3460 \mathrm{G}>\mathrm{A}$, and $14484 \mathrm{~T}>\mathrm{C}$ point mutations in $M T$ ND4, MT-ND1, and MT-ND6. The unusually high mitochondrial load of RGCs makes them particularly sensitive to these mutations. Patients with LHON may be prescribed ubiquinone (a component of RC3) or idebenone, a ubiquinone analogue with enhanced bioavailability to act downstream of RC1. The challenge of accessing the inner mitochondrial membrane with gene therapy for LHON, and other mitochondrial diseases, may be overcome by incorporation of a specific mitochondrion-targeting sequence (MTS)
\end{abstract}

Enhanced Digital Features To view enhanced digital features for this article go to https://doi.org/10.6084/ m9.figshare.9912680.

C. Karaarslan $(\bowtie)$

World Eye Hospital, Adana, Turkey

e-mail: aktifgoz@hotmail.com that enables allotropic expression of a nucleustranscribed ND4 transgene. Because LHON penetrance is incomplete among carriers of the aforementioned mutations, identification of environmental factors, such as heavy smoking, that interact with genetics in the phenotypic expression of LHON may be helpful toward preventing or delaying disease development. LHON has become a model for mitochondrial and neurogenerative diseases owing to it having a clearly identified genetic cause and its early onset and rapid progression characteristics. Hence, LHON studies and genetic treatment advances may inform research of other diseases.

Keywords: Genes; Gene therapy; Leber's hereditary optic neuropathy; Mutation

\section{INTRODUCTION}

Leber's hereditary optic neuropathy (LHON; OMIM 535000) is a disease characterized by bilateral progressive vision loss that was first described by German ophthalmologist Theodore Leber in 1871 [1]. Currently, LHON is one of the most common inherited optic neuropathies with a prevalence of 1 in 30,000 [2]. It is diagnosed most frequently in teenage boys and young men (15-35 years old), which account for $80 \%$ of new LHON cases, but can occur in individuals of all ages, including 
children and the elderly [3], with the age of onset tending to be higher in female individuals (initially, affected patients present with painless visual loss-principally central or centrocecal scotoma-either in both eyes simultaneously (one in four cases) or sequentially with the second eye showing symptoms within 8 weeks of the first (three in four cases)); unilateral LHON is very rare [4]. Disease progression is rapid in the acute phase, with visual acuity being diminished to $2 / 20$ or less within 5-6 weeks of the onset of symptoms [5]. Some spontaneous recovery of vision has been observed, though rarely [6].

With respect to clinical pathological development, in most cases, fundus examination of LHON-affected eyes reveals several characteristic signs, including circumpapillary telangiectatic microangiopathy, blood vessel tortuosity, and edema of the retinal nerve fiber layer and optic disc [7]. However, some $20-25 \%$ of patients with LHON present with a normal-appearing fundus in the acute stage [8]. In the chronic stage, optic atrophy due to the loss of retinal ganglion cells (RGCs) can be observed [9]. However, even with recovery, central visual field defects and dyschromatopsia can be permanent and most patients with LHON end up legally blind [10].

Etiologically, LHON has been confirmed to be a maternally inherited mitochondrial disorder, though about one in three cases appears to be sporadic with no definitive family history [9]. As elaborated below, point mutations in mitochondrial DNA (mtDNA) genes that encode components of respiratory complex (RC) 1 (also known as NADH:ubiquinone oxidoreductase) have been associated with LHON. Loss of fully functioning RC1 leads to reduced ATP production via the mitochondrial electron transport chain and increased oxidative stress in RGCs [11]. This article is based on previously conducted studies and does not contain any studies with animals performed by the author.

\section{MITOCHONDRIAL GENE MUTATIONS AND RGC DYSFUNCTION}

The prognosis of patients with LHON tends to differ depending upon the underlying pathogenic mutation involved. All LHONcausing mutations are in mtDNA, and thus identified by their nucleotide positions within mtDNA. The most common LHON-causing mutations are a G-to-A substitution at nucleotide 11778, a G-to-A substitution at nucleotide 3460, and a T-to-C substitution at nucleotide 14484 within the mitochondrial genes ND4, ND1, and ND6 genes, respectively. ND4, ND1, and ND6 encode the NADH dehydrogenase 4, 1 , and 6 proteins, respectively, within RC1 of the oxidative phosphorylation chain in mitochondria. These 11778G $>A, \quad 3460 \mathrm{G}>\mathrm{A}$, and $14484 \mathrm{~T}>\mathrm{C}$ mutations have been reported to be responsible for $50-70 \%, 8-25 \%, 10-15 \%$ of LHON cases, respectively [12]. Notably, the $11778 \mathrm{G}>\mathrm{A}$ mutation in ND4 causes the most severe LHON-related visual loss, whereas the $3460 \mathrm{G}>\mathrm{A}$ mutation has a relatively moderate prognosis [13].

Because mitochondria are the primary sources of cellular energy, the mitochondrial dysfunction associated with primary mitochondrial diseases, such as LHON, can have severe effects on cell growth and the synthesis of critical biological molecules, such as heme, neurotransmitters, hormones, and even the nucleotide bases that constitute DNA and RNA molecules. Neurodegeneration, seizures, myopathy, cardiomyopathy, deafness, optic nerve atrophy, and developmental delays are common symptoms of mitochondrial disorders [14].

Neurons in the retina, especially within the optic disc area, have high numbers of mitochondria. This cellular characteristic makes them particularly sensitive to mitochondrial dysfunction and associated impairment of axonal transport; within the retina, the prelaminar and intralaminar regions have the greatest concentrations of mitochondria [15]. Accordingly, patients with LHON tend to show selective loss of RGCs in the temporo-central portion of the optic disc. Concomitantly, oligodendrocytes insulating the optic nerves become unable to produce sufficient ATP and, as a consequence, exhibit degeneration, shrinkage, chromatin condensation, chromosomal DNA fragmentation, and, ultimately, cell death [16]. 


\section{GENE-ENVIRONMENT INTERACTIONS IN LHON}

For unknown reasons, LHON penetrance is incomplete among carriers of the mtDNA gene mutations that cause LHON, with only about half of male and about a tenth of female individuals with an offending mutation developing LHON in their lifetimes [17]. It has been suggested that mtDNA-haplogroup background may explain, at least in part, LHON penetrance variance across individuals [18]. However, there are reported cases of identical twin pairs in which one twin had developed LHON whilst the other twin remained unaffected thus far [19]. The existence of such twin discordance together with the incomplete penetrance of LHON among causative mutation carriers in the population suggests that although LHON has a well-defined genetic basis, it is also a multifactorial disease with environmental factors that trigger its pathogenesis in genetically vulnerable individuals [20].

When surveyed regarding notable life events or exposures that preceded and appeared to trigger vision loss, patients with LHON cited psychological stress, major physical trauma, malnutrition, and chemotherapy [21]. Apart from a singular putative trigger experience, tobacco smoking and alcohol use have been explored as potential environmental factors favoring LHON pathogenesis, with the former producing more convincing and consistent effects than the latter. In particular, binary logistic regression modeling identified heavy smoking (but not light smoking, light drinking, or heavy drinking) as a significant predictor of LHON development. It could be that smokers accumulate additional mtDNA mutations that act additively with the primary LHON-causing mutation, further compromising mitochondrial resilience [22]. However, an aggravating effect of smoking on LHON pathogenesis cannot explain the development of LHON in nonsmokers and young children with limited potential environmental triggers [23].

Considering gender and smoking together, Kirkman and colleagues [21] found that more than nine-tenths of heavy smoking male LHON mutation carriers developed the disease compared to two-thirds of non-smoking male carriers and only one-third of smoking female carriers. This gender bias may be consequent to a protective effect of having two $\mathrm{X}$ chromosomes protecting female individuals from gene variants on the $X$ chromosome that increase susceptibility to LHON pathogenesis [24].

\section{LHON TREATMENT}

\section{Pharmacological Treatment}

Whilst there is not yet a curative or high-efficacy treatment for LHON [25], patients with mitochondrial diseases, including LHON, are commonly prescribed ubiquinone (also known as coenzyme Q10), a component of RC3 [26]. A molecular analogue of ubiquinone with enhanced bioavailability called idebenone has been recommended [27]. Cree and colleagues [28] reported that significant recovery of vision could be achieved, at least temporarily, with idebenone [28]. Subsequently, in a RHODOS (Rescue of Hereditary Optic Disease Outpatient Study) randomized and placebo-controlled, double-blind trial with 85 patients, the group given high-dose idebenone $(900 \mathrm{mg})$ for 24 weeks had better vision than placebo controls without any signs of adverse drug reactions. Patients with early-stage disease appeared to benefit the most [29]. These molecules are thought to counter RC1 deficiency by acting downstream of RC1, effectively bypassing it, to augment ATP production. In conclusion, these studies presented by researchers from all over the world suggest that idebenone treatment, especially in patients with acute LHON, may improve visual recovery [27-30].

\section{Gene Therapy}

There is optimism that LHON may be cured, prevented, or at least reduced in severity with gene therapies. The goal of gene therapy for LHON, and other mitochondrial diseases, is to rescue mitochondrial function to an extent that is sufficient to at least relieve the symptoms of, 
if not cure, the target disease by supplementing intact/wild-type alleles of the dysfunctional gene above the clinical threshold for a normal physiological phenotype.

The site of the mutated mtDNA genes underlying LHON, however, is within the mitochondrial inner membrane. The mitochondrial chromosome itself is relatively simple at only 16,569 base pairs long and encoding 13 mRNAs and their associated non-coding promoter regions, as well as 2 rRNAs and 22 tRNAs for local intra-mitochondrial translation. The advantage of the genetic simplicity of mtDNA, however, is countered by its inaccessibility deep within the double-membraned mitochondrion organelle, which, normally, allows the passage of only very small molecules, such as ATP and proteins that are smaller than $10 \mathrm{kDa}$ [31]. The relative impermeability of mitochondria creates an access challenge with well-established gene delivery vectors, such as adenovirus vectors (AVVs).

To deliver a therapeutic gene product to mitochondria, one must first achieve cellular endocytosis, wherein the gene product is taken up by body cells. Secondly, one must avoid destruction by endosomes in the cytoplasm. Finally, in the most challenging problem facing researchers developing mitochondrial gene therapy approaches, one must devise a mechanism by which the gene product is made to cross into the internal space of the mitochondrion. Because nucleic acids are hydrophilic, even very small naked DNA molecules do not cross the mitochondrial membrane unaided. Given these challenges, simple physical methods that can achieve gene transfer from the extracellular space into the intracellular space, such as penetration by hydrodynamic force or bombardment, have poor efficacy for achieving targeted transfer to mitochondria within intact cells [32].

There are a substantial variety of chemical approaches to mitochondrion-targeted gene delivery, and the approaches continue to evolve as researchers work to improve efficacy and specificity of delivery. Unfortunately, chemical approaches that rely on membrane destabilization to achieve transfer tend to be highly cytotoxic and thus not readily translatable to clinical applications [33]. Thus, there has been substantial development of reduced-cytotoxicity cationic surfactants to carry DNA to and into mitochondria [34, 35]. Most simply, DNA plasmids have been conjugated directly to lipophilic rhodamine carrier molecules [36]. Efforts to improve the mitochondrion-targeting efficiency, carrier stability, and toxicity profiles of DNA carriers are ongoing. Liposome-type DNAcarrying vesicles, most notably vesicles produced from modified forms of dequalinium, have been shown to accumulate in mitochondria [37-40]. There has been interest in the potential benefits of conjugating liposomes with triphenylphosphonium cation-containing molecules to improve mitochondrion targeting of carriers [41].

There are several highly promising current lines of research aimed at developing safe and effective biological strategies for reconstituting ND4-deficient mitochondria in RGCs with wildtype ND4, with the long-term goal of producing a cure for LHON. Biological approaches to mtDNA delivery with ever-increasing eloquence are being developed. Some research groups have incorporated mitochondrial targeting signal (MTS) peptide tags used by cells in their strategies to deliver molecules to mitochondria, such as in MTS-conjugated peptide nucleic acid carriers [42], MTS-conjugated lysine/histidine peptide carriers [43, 44], and MTS-conjugated AVV carriers [45]. This approach has been reported to provide dramatic protection from RGC loss in animal models. Notably, Yu et al. [46] demonstrated long-term expression of ND4, mutations of which are responsible for most cases of LHON, in mouse cells transfected with human ND4 via an AVV carrier in which the viral capsid VP2 had been modified to include an MTS [46].

Several research groups have worked around the mitochondrion-targeting problem by focusing on getting the ND4 product into mitochondria after expression rather than the whole vector. This approach, known as allotopic expression, is based on the premise that ND4 transcribed in the nucleus will be translated in the cytoplasm and then delivered to mitochondria by way of a translated MTS in the same manner that intrinsic mitochondrial 
proteins encoded by nuclear genes are delivered to mitochondria. Koilkonda et al. [47] optimized a serotype-2 AVV (AVV2) carrier for intravitreal delivery of wild-type ND4 with the MTS nucleotide sequence from the ATP1 gene and demonstrated efficacy across a variety of experimental systems, including an in vivo mouse model, an in vivo macaque model, and ex vivo human eye model (eyes removed because of cancer). In mice, they were able to achieve allotopic mitochondrial expression of ND4 in 85\% of RGCs within a week of injection. Moreover, follow-up imaging, physiological, and histological assessments demonstrated that their gene therapy strategy led to attenuation of experimental LHON model pathogenesis with respect to RGC loss, local ATP production loss, vision loss, and optic nerve atrophy [47]. In primates, they demonstrated that their AAV2-ND4 delivery system was well tolerated. In human eyes, they demonstrated an accumulation of allotopic ND4 protein in the mitochondria of in situ human RGCs [48].

Employing a similar strategy, Cwerman-Thibault et al. [49] developed an AAV2/2-ND4 delivery system using the MTS from COX10. They demonstrated efficient incorporation of ND4 protein into RC1 of RGCs in 8-week-old rats. Moreover, AAV2/2-ND4-treated LHONmodel rats exhibited attenuated RGC degradation and preservation of visual function [49].

Following the aforementioned promising results, Feuer and colleagues [50] conducted a phase I safety trial for allotopic AAV2-ND4 gene therapy in human patients who were legally blind due to LHON caused by the $11778 \mathrm{G}>\mathrm{A}$ ND4 mutation. Of the five patients in the study, one experienced temporary minor adverse effects in the injected eye, including increased intraocular pressure and subconjunctival hemorrhage. No negative outcomes, such as further vision loss or major adverse events, were observed. Ninety days after the procedure, best corrected visual acuity (BCVA) remained unchanged in three patients, but had improved significantly in two patients [50].

Meanwhile, researchers in the Li laboratory conducted a prospective gene therapy study in nine patients diagnosed with LHON, also due to the $11778 \mathrm{G}>\mathrm{A}$ ND4 mutation, with no spontaneous improvements in BCVA during the year preceding the study. Nine months after intravitreal injection of AAV2-ND4 with the COX10 MTS, significant improvements in BCVA were observed in six out of nine patients. Interestingly, patients who experienced improved BCVA in the injected eye often experienced some concurrent improvement in the contralateral non-injected eye. They conducted an accompanying experiment in mice to examine this phenomenon of contralateral improvement, the findings of which suggested it may be mediated via inter-communication at the optic chiasm [51].

In a subsequent 36-month follow-up study of the same nine patients, no adverse outcomes were found in any of the patients. One of the patients underwent the same procedure in the other eye during follow-up, and was thus analyzed separately. Of the remaining eight patients, four experienced a significant improvement in BCVA from baseline to the 36-month follow-up time point. These four patients had a similar age of onset, LHON illness duration, and retinal nerve fiber layer thickness (both at baseline and at the 36-month time point) as the four patients who did not experience a significant improvement in BCVA, indicating that these factors could not explain patient outcomes. Temporary visual field improvements were seen in four out of eight patients, peaking between 3 and 6 months after the treatment, whereas two patients continued to show progressive visual field improvement through the 36-month time point [52].

Moreover, GenSight Biologics, Paris, France, in three phase 3 studies on LHON demonstrated that rAAV2/2-ND4 is safe and well tolerated 2 years after a single unilateral intravitreal administration. Even so, some patients experienced early improvement on visual acuity, color vision, and contrast sensitivity in the treated eye. The most common adverse events during these studies were mild anterior chamber or vitreous inflammation and moderate intraocular pressure elevation. All ocular side effects were solved with standard therapy and no visual sequelae occurred [53-55]. 


\section{LHON AS A MODEL DISEASE}

LHON has become a research model for mitochondrial diseases in general owing to its wellestablished genetic etiology and course of progression. Thus, findings obtained in LHON studies may also be important for other mitochondrial diseases. Additionally, the neural damage that occurs in LHON resembles that in other neurodegenerative conditions, including Parkinson disease and Alzheimer disease, which affect millions of people worldwide. Among neurodegenerative diseases, which are often slowly progressing, LHON has a relatively early onset and unusually rapid progression. These characteristics facilitate its use as a model disease. Hence, ongoing and future studies examining the efficacy of neuroprotective agents and gene therapy for suppressing or reversing LHON are likely to inform research examining other mitochondrial and neurodegenerative diseases.

\section{CONCLUSION AND FUTURE DIRECTIONS}

Basic research exploring and developing gene therapy approaches has made substantial progress, bringing us into an era of emergent clinical genetic therapies. Genetic-based diseases of the eyes are an attractive realm within which to develop gene therapy methods owing to their small size and straightforward accessibility. LHON in particular is an attractive target disease for gene therapy because the mutations underlying its pathogenesis are known and its etiology, which is well characterized, involves early onset and rapid progression. Meanwhile, substantial further work is needed to clarify how LHON-causing gene mutations interact with other intrinsic individual biological factors (e.g., gender, haplotype, gene-gene interactions) to determine penetrance and development of the disease in genetically susceptible individuals. Moreover, there is a similar need for continued research aimed at elucidating the environmental factors (e.g., heavy smoking, exposure to toxins, trauma) that trigger phenotypic visual loss in genetically susceptible individuals. A better understanding of how such factors contribute to LHON pathogenesis may provide critical information for preventing LHON, delaying LHON onset, and attenuating LHON severity.

There have been major advances in overcoming the challenge inherent in delivering gene therapy products to mitochondria, and solutions to this accessibility problem that are developed and explored in LHON research can be transferred to other mitochondrial genetic diseases, making LHON a particularly technically useful disease model. MTS tags on gene carrier vectors or appended to the gene being injected for subsequent translation have shown great promise for overcoming the mitochondrion access challenge. Importantly, the data available to date indicate that allotopic expression of ND4 can be achieved safely and can yield clinical improvements in vision. Large multicenter randomized controlled trials are needed to confirm and extend recent encouraging findings in small cohorts.

\section{ACKNOWLEDGEMENTS}

Funding. No funding or sponsorship was received for this study or publication of this article. The Rapid Service Fee was funded by the author.

Medical Writing and/or Editorial Assistance. This manuscript was prepared with professional manuscript editing support from Dr. Ann Power Smith at Write Science Right.

Authorship. The named author meets the International Committee of Medical Journal Editors (ICMJE) criteria for authorship for this article, takes responsibility for the integrity of the work as a whole, and has given his approval for this version to be published.

Disclosures. Cuneyt Karaarslan has nothing to disclose.

Compliance with Ethics GuidelinesThis article is based on previously conducted studies and does not contain any studies with human 
participants or animals performed by the author.

Open Access. This article is distributed under the terms of the Creative Commons Attribution-NonCommercial 4.0 International License (http://creativecommons.org/licenses/ by-nc/4.0/), which permits any noncommercial use, distribution, and reproduction in any medium, provided you give appropriate credit to the original author(s) and the source, provide a link to the Creative Commons license, and indicate if changes were made.

\section{REFERENCES}

1. Leber T. Ueber hereditaere und congenital angelegte sehnervenleiden. Graefes Arch Clin Exp Ophthalmol. 1871;17(2):249-91.

2. Man PY, Griffiths PG, Brown DT. The epidemiology of Leber hereditary optic neuropathy in the North East of England. Am J Hum Genet. 2003;72(2):333-9.

3. Yu-Wai-Man P, Griffiths PG, Hudson G, Chinnery PF. Inherited mitochondrial optic neuropathies. J Med Genet. 2009;46(3):145-58.

4. Puomila A, Hamalainen P, Kivioja S, Savontaus ML, Koivumaki S, Huoponen K. Epidemiology and penetrance of Leber hereditary optic neuropathy in Finland. Eur J Hum Genet. 2007;15(10):1079-89.

5. Fraser JA, Biousse V, Newman NJ. The neuro-ophthalmology of mitochondrial disease. Surv Ophthalmol. 2010;55(4):299-334.

6. Ghelli A, Zanna C, Porcelli AM, Schapira AH, Martinuzzi A, Carelli V. Leber's hereditary optic neuropathy (LHON) pathogenic mutations induce mitochondrial-dependent apoptotic death in transmitochondrial cells incubated with galactose medium. J Biol Chem. 2003;278(6):4145-50.

7. Gropman A, Chen TJ, Perng CL, Krasnewich D, Chernoff E, Tifft C. Variable clinical manifestation of homoplasmic G14459A mitochondrial DNA mutation. Am J Med Genet A. 2004;124A(4): $377-82$.

8. Fraser JA, Ross-Cisneros CPF. Pathoimmunologic findings which are important in the etiology of mitochondrial DNA disease in adults. Ann Neurol. 2008;63(1):35-9.
9. Spruijt L, Smeets HJ, Hendrickx A, Bettink-Remeijer MW, Maat-Kievit A, Schoonderwoerd KC. A MELASassociated ND1 mutation causing Leber hereditary optic neuropathy and spastic dystonia. Arch Neurol. 2007;64(6):890-3.

10. Carelli V, Ross-Cisneros FN, Sadun AA. Mitochondrial dysfunction as a cause of optic neuropathies. Prog Retin Eye Res. 2004;23(1):53-89.

11. Valentino ML, Barboni P, Ghelli A, Bucchi L, Rengo C, Achilli A. The ND1 gene of complex I is a mutational hot spot for Leber's hereditary optic neuropathy. Ann Neurol. 2004;56(5):631-41.

12. La Morgia C, Ross-Cisneros FN, Sadun AA, et al. Melanopsin retinal ganglion cells are resistant to neurodegenerationin mitochondrial optic neuropathies. Brain. 2010;133(Pt 8):2426-38.

13. Battisti C, Formichi P, Cardaioli E, Bianchi S, Mangiavacchi P, Tripodi SA. Cell response to oxidative stress induced apoptosis in patients with Leber's hereditary optic neuropathy. J Neurol Neurosurg Psychiatry. 2004;75(12):1731-6.

14. Wang JY, Gu YS, Wang J, Tong Y. Oxidative stress in Chinese patients with Leber's hereditary optic neuropathy. J Int Med Res. 2008;36(3):544-50.

15. McFarland R, Chinnery PF, Blakely EL, et al. Homoplasmy, heteroplasmy, and mitochondrial dystonia. Neurology. 2007;69(9):911-6.

16. Barron MJ, Griffiths P, Turnbull DM, Bates D, Nichols P. The distributions of mitochondria and sodium channels reflect the specific energy requirements and conduction properties of the human optic nerve head. $\mathrm{Br} \mathrm{J}$ Ophthalmol. 2004;88(2):286-90.

17. Seedorff T. The inheritance of Leber's disease. A genealogical follow-up study. Acta Ophthalmol. 1985;63(2):135-45.

18. Hudson G, Carelli V, Spruijt L, et al. Clinical expression of Leber hereditary optic neuropathy is affected by the mitochondrial DNA-haplogroup background. Am J Hum Genet. 2007;81(2):228-33.

19. Johns DR, Smith KH, Miller NR, Sulewski ME, Bias WB. Identical twins who are discordant for Leber's hereditary optic neuropathy. Arch Ophthalmol. 1993;111(11):1491-4.

20. Biousse $\mathrm{V}$, Browne MD, Newman NJ, et al. De novo 14484 mitochondrial DNA mutation in monozygotic twins discordant for Leber's hereditary optic neuropathy. Neurology. 1997;49(4):1136-8. 
21. Kirkman MA, Yu-Wai-Man P, Korsten A. Gene environment interactions in Leber hereditary optic neuropathy. Brain. 2009;132(Pt 9):2317-26.

22. Chalmers RM, Harding AE. A case-control study of Leber's hereditary optic neuropathy. Brain. 1996;119(Pt 5):1481-6.

23. Tsao K, Aitken PA, Johns DR. Smoking as an aetiological factor in a pedigree with Leber's hereditary optic neuropathy. Br J Ophthalmol. 1999;83(5): 577-81.

24. Shankar SP, Fingert JH, Carelli V, et al. Evidence for a novel X-linked modifier locus for Leber hereditary optic neuropathy. Ophthalmic Genet. 2008;29(1):17-24.

25. Yu-Wai-Man P, Griffiths PG, Chinnery PF. Mitochondrial optic neuropathies-disease mechanisms and therapeutic strategies. Prog Retin Eye Res. 2011;30(2):81-114.

26. Haefeli RH, Erb M, Gemperli AC. NQO1-dependent redox cycling of idebenone: effects on cellular redox potential and energy levels. PLoS One. 2011;6(3):e17963.

27. Klopstock K, Yu-Wai-Man P, Dimitriadis K, et al. A randomized placebo controlled trial of idebenone in Leber's hereditary optic neuropathy. Brain. 2011;134(9):2677-86.

28. Cree LM, Samuels DC, Chinnery PF. The inheritance of pathogenic mitochondrial DNA mutations. Biochim Biophys Acta. 2009;1792(12):1097-102.

29. Giordano C, Montopoli M, Perli E. Oestrogens ameliorate mitochondrial dysfunction in Leber's hereditary optic neuropathy. Brain. 2011;134(Pt 1):220-34.

30. Qi XP, Sun L, Hauswirth WW. Use of mitochondria antioxidant defenses for rescue of cells with a Leber hereditary optic neuropathy-causing mutation. Arch Ophthalmol. 2007;125(2):268-72.

31. Jang Y, Lim K. Recent advances in mitochondriatargeted gene delivery. Molecules. 2018;23(9): E2316.

32. Ma XC, Wang XB, Zhou M, Fei HA. Mitochondriatargeting gold-peptide nanoassembly for enhanced cancer-cell killing. Adv Healthc Mater. 2013;2(12): $1638-43$.

33. Boddapati SV, D'Souza GG, Erdogan S, Torchilin VP, Weissig V. Organelle-targeted nanocarriers: specific delivery of liposomal ceramide to mitochondria enhances its cytotoxicity in vitro and in vivo. Nano Lett. 2008;8(8):2559-63.
34. Wang WX, He YF, Shang YZ, Liu HL. Interaction between the gemini surfactant (12-6-12) and DNA. Acta Physico-Chim Sin. 2011;27(1):156-62.

35. Cardoso AM, Faneca H, Almeida JA, et al. Gemini surfactant dimethylene-1,2-bis(tetradecyldimethylammonium bromide)-based gene vectors: a biophysical approach to transfection efficiency. Biochim Biophys Acta. 2011;1808(1):341-51.

36. Salvado R, Sousa F, Queiroz J, Costa D. Development of mitochondrial targeting plasmid DNA nanoparticles: characterization and in vitro studies. Colloid Surf A. 2015;480:287-95.

37. Futaki S, Ohashi W, Suzuki T, et al. Stearylated arginine-rich peptides: a new class of transfection systems. Bioconjug Chem. 2001;12(6):1005-11.

38. D'Souza GGM, Rammohan R, Cheng SM, Torchilin VP, Weissig V. DQAsome-mediated delivery of plasmid DNA toward mitochondria in living cells. J Control Release. 2003;92(1-2):189-97.

39. Bae Y, Jung MK, Song SJ, et al. Functional nanosome for enhanced mitochondria-targeted gene delivery and expression. Mitochondrion. 2017;37: 27-40.

40. Yamada Y, Ishikawa T, Harashima H. Validation of the use of an artificial mitochondrial reporter DNA vector containing a cytomegalovirus promoter for mitochondrial transgene expression. Biomaterials. 2017;136:56-66.

41. Biswas S, Dodwadkar NS, Deshpande PP, Torchilin VP. Liposomes loaded with paclitaxel and modified with novel triphenylphosphonium-PEG-PE conjugate possess low toxicity, target mitochondria and demonstrate enhanced antitumor effects in vitro and in vivo. J Control Release. 2012;159(3): 393-402.

42. Santos JL, Pandita D, Rodrigues J, et al. Receptormediated gene delivery using PAMAM dendrimers conjugated with peptides recognized by mesenchymal stem cells. Mol Pharm. 2010;7(3):763-74.

43. Midoux P, Pichon C, Yaouanc JJ, Jaffres PA. Chemical vectors for gene delivery: a current review on polymers, peptides and lipids containing histidine or imidazole as nucleic acids carriers. Br J Pharmacol. 2009;157(2):166-78.

44. Chuah JA, Matsugami A, Hayashi F, Numata K. Selfassembled peptide-based system for mitochondrialtargeted gene delivery: functional and structural insights. Biomacromolecules. 2016;17(11):3547-57.

45. Daya S, Berns KI. Gene therapy using adeno-associated virus vectors. Clin Microbiol Rev. 2008;21(4):583-93. 
46. Yu H, Koilkonda RD, Chou TH, et al. Gene delivery to mitochondria by targeting modified adenoassociated virus suppresses Leber's hereditary optic neuropathy in a mouse model. Proc Natl Acad Sci USA. 2012;109(20):E1238-47.

47. Koilkonda RD, Yu H, Chou TH, et al. Safety and effects of the vector for the Leber hereditary optic neuropathy gene therapy clinical trial. JAMA Ophthalmol. 2014;132(4):409-20.

48. Koilkonda $\mathrm{R}, \mathrm{Yu} \mathrm{H}$, Talla $\mathrm{V}$, et al. LHON gene therapy vector prevents visual loss and optic neuropathy induced by G11778A mutant mitochondrial DNA: biodistribution and toxicology profile. Invest Ophthalmol Vis Sci. 2014;55(12):7739-53.

49. Cwerman-Thibault H, Augustin S, Lechauve C, et al. Nuclear expression of mitochondrial ND4 leads to the protein assembling in complex I and prevents optic atrophy and visual loss. Mol Ther Methods Clin Dev. 2015;2:15003.

50. Feuer WJ, Schiffman JC, Davis JL, et al. Gene therapy for Leber hereditary optic neuropathy: initial results. Ophthalmology. 2016;123(3):558-70.

51. Wan X, Pei H, Zhao MJ, et al. Efficacy and safety of rAAV2-ND4 treatment for Leber's hereditary optic neuropathy. Sci Rep. 2016;6:21587.
52. Yang S, Ma SQ, Wan X, et al. Long-term outcomes of gene therapy for the treatment of Leber's hereditary optic neuropathy. EBio Med. 2016;10:258-68.

53. Vignal C, Uretsky S, Thomasson N, et al. Recombinant AAV2 containing the wild type ND4 gene (RAVV2/2-ND4) is an experimental gene therapy for vision loss in LHON due to the ND4 mitochondrial mutation: phase I/IIa safety investigation results and upcoming pivotal phase III efficacy studies. In: North-American Neuro-Ophthalmology Society 42nd annual meeting, February 27-March 3, 2016; Tucson, Arizona.

54. Vignal S, Uretsky S, Fitoussi S, et al. Safety of rAVV2/2-ND4 gene therapy for Leber's hereditary optic neuropathy. Ophthalmology. 2018;125(6): 945-7.

55. Thomasson N, Cwerman-Thibault H, Augustin S, et al. Safety, local tolerability and biodistribution of GS010 (AAV2/2-ND4), a gene therapy medicinal product in development for Leber's hereditary optic neuropathy (LHON). ARVO Annual Meeting, 2014. Invest Ophthalmol Vis Sci. 2014;55:3340. 